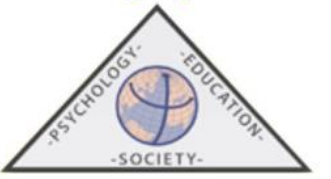

\title{
Acompañamiento profesional para la reinserción e inclusión social de mujeres en semilibertad
}

\author{
Ana Amaro Agudo ${ }^{1}$, Clotilde Berzosa Sáez ${ }^{1,2}$, Ana Alcázar-Campos ${ }^{1}$ y María del Mar García- \\ Vita $^{3}$ \\ ${ }^{1}$ Universidad de Granada \\ ${ }^{2}$ CIS Granada SGIP \\ ${ }^{3}$ Universidad de Almería \\ Recibido el 08 de mayo de 2020. Aceptado el 20 de abril de 2021
}

\begin{abstract}
RESUMEN: El presente artículo tiene como objetivo describir el tipo de acompañamiento institucional recibido por la población femenina en el medio abierto del Sistema Penitenciario Español, como ayuda a la inclusión social y la reinserción. Este trabajo se enmarca dentro del proyecto de investigación $\mathrm{I}+\mathrm{D}+\mathrm{I}$ "Procesos de reinserción y acompañamiento a mujeres en semilibertad (ref. EDU2016-79322-R) cuya metodología ha empleado métodos cualitativos y cuantitativos, de forma complementaria, aplicados a los 310 cuestionarios mixtos y a las 67 entrevistas semiestructuradas, diseñados ad hoc, en 31 centros penitenciarios que representan los distintos recursos de cumplimiento de condena en medio abierto. Se ha realizado un análisis descriptivo de los resultados de cinco preguntas del cuestionario de mujeres relativo al acompañamiento, tipo y profesionales implicados en el mismo. Los resultados obtenidos evidencian que para las mujeres del estudio es necesario el acompañamiento para un $85.2 \%$ a nivel general, recibiéndolo un $31.3 \%$. El número de profesionales que participan en el mismo es por lo general importante siendo la figura del trabajador/a social, el/la educador/a y el/la psicólogo/a con el 54.2\%, $46.8 \%$ y $32.9 \%$ respectivamente los más solicitados. El acompañamiento socioeducativo facilita la identificación de un proceso de cambio personal, la reflexión, el apoyo y el empoderamiento personal.
\end{abstract}

Palabras clave: asesoramiento, equipo técnico, género, prisión.

\section{Professional accompaniment for the reinsertation and social inclusion of social inclusion in semi- freedom}

\begin{abstract}
The objective of this article is to describe the type of institutional support received by the female population in the open environment of the Spanish Penitentiary System, as an aid to social inclusion and reintegration. This research is part of the project $\mathrm{I}+\mathrm{D}+\mathrm{I}$ " Processes of reintegration and accompaniment of women in semi-freedom (ref. EDU2016-79322-R) using a qualitative and quantitative methodology applied to the 310 mixed questionnaires and 67 semi-structured interviews, designed ad hoc, in 31 penitentiary centers representing the different resources for serving an open sentence. A descriptive analysis of the results of five questions of the women's questionnaire regarding accompaniment, type and professionals involved in it was carried out. The results obtained show that for the women in the study, accompaniment is necessary for $85.2 \%$ at a general level, receiving it by $31.3 \%$. The number of professionals who participate in it is generally important, being the figure of the social worker, the educator and the psychologist with $54.2 \%, 46.8 \%$ and $32.9 \%$ respectively the most requested. Socioeducational accompaniment facilitates the identification of a process of personal change, reflection, support and personal empowerment.
\end{abstract}

Keywords: counseling, technical team, gender, prison.

Correspondencia: María del Mar García-Vita. Universidad de Almería. Carretera Sacramento s/n, Edifico Central, despacho 0.842, 04120, La Cañada de San Urbano (Almería). Email: margvita@ual.es 


\section{Introducción}

Los centros penitenciarios se enfrentan al reto de conciliar objetivos contradictorios: el mantenimiento del orden y la seguridad, y el tratamiento rehabilitador (Ayuso, 2001; Redondo, 2004; Redondo et al., 2007). En ocasiones, ambos entran en conflicto debido a que la consecución de los objetivos de tratamiento colisiona con un medio en el que las actividades humanas -hasta las más básicas- están reglamentadas y controladas (Ayuso, 2001). Debido a esta dualidad existen figuras profesionales propias del régimen penitenciario y aquellas propias del tratamiento penitenciario, siendo necesaria una coordinación entre ambas (Redondo et al., 2007). En este trabajo queremos poner en valor la vertiente socioeducativa del tratamiento penitenciario, que se hace patente desde la óptica del propio sistema, ya que su función prioritaria, según la legislación, debe ser la de la reeducación y rehabilitación de los sujetos (García-Vita, 2016). Desde las instituciones penitenciarias se reconoce este hecho y actualmente se busca alejarse de la acepción más terapéutica y clínica del tratamiento penitenciario y acercarse a posturas más asistenciales, formativas, sociolaborales o socioculturales de intervención psicosocial y tratamiento específico (Añaños-Bedriñana y Yagüe, 2013). Una estrategia clave para ello es el acompañamiento que, desde la institución, los y las profesionales y las entidades colaboradoras externas realizan, sobre todo en la fase de retorno a la vida en libertad.

El objetivo de este artículo es describir el tipo de acompañamiento institucional prestado a la población femenina en el medio abierto del Sistema Penitenciario Español, cómo este ayuda a la inclusión social y la reinserción, qué tipos hay y cuáles son los y las profesionales que intervienen. Y, concretamente, valorar si existe acompañamiento en prisión, el tipo de asesoramiento y profesionales a los/as que acuden para realizar ese proceso de acompañamiento. Además de conocer la opinión de los/as profesionales sobre el proceso de acompañamiento y el tipo.

\section{Profesionales, organización penitenciaria y perspectiva socioeducativa}

Los establecimientos penitenciarios se constituyen como un núcleo autosuficiente donde desarrollan su labor multitud de profesionales, estructurándose en cuerpos y categorías profesionales y garantizándose la cobertura de diversas especialidades (Secretaría General de Instituciones Penitenciarias, 2014). Dichos profesionales se encuentran organizados en grupos según las distintas áreas de trabajo (intervención, sanitaria, servicios y mantenimiento). Además, existe una estructura en órganos de gobierno según el Reglamento Penitenciario de 1996: Junta de Dirección, Junta de Tratamiento vinculado al Equipo Técnico, Comisión Disciplinaria y Junta Económica-Administrativa; y una articulación en unidades técnico-funcionales de diferente rango administrativo y en una estructura constituida por centros penitenciarios ordinarios y los centros de inserción social. Entre estos espacios profesionales hay que destacar la importancia del Equipo Técnico, puesto que son sus profesionales quienes trabajan directamente con los internos e internas, ejecutando los planes y acciones previstas para apoyarles. En contraste, la Junta de Tratamiento se ocupa principalmente de la toma de decisiones y de establecer esas pautas de acción a ejecutar por el Equipo Técnico (García-Vita y Melendro, 2013; Secretaría General de Instituciones Penitenciarias, 2014).

Varias son las disciplinas que se han ocupado de colaborar y llevar a cabo todo lo relativo al tratamiento, entre ellas destacan la Psicología o la Criminología, y, desde hace pocas décadas, la Pedagogía y la Educación Social. Esto ha llevado a que se haya definido la Educación Social Penitenciaria (Añaños, 2010; Del Pozo y Añaños, 2013) como una disciplina liberadora que aboga por que la educación es posible en contextos cerrados, siendo un campo emergente dentro de las áreas de la Pedagogía y la Educación Social. Ésta se ve modificada y fortalecida en el contexto penitenciario a partir de la circular de la Dirección General de Instituciones Penitenciarias del año 2006, que imbrica en la 
Subdirección General de Tratamiento y Gestión Penitenciaria lo referente a las actividades educativas, deportivas, ocupacionales y culturales (Del Pozo y Añaños, 2013). Al mismo tiempo, se impulsan los equipos multidisciplinares y la aplicación de programas educativos individuales (PEI) y los grupales (PEG), donde la dimensión crítica, autónoma y emancipadora de las personas se erige como fundamental y donde adquiere un mayor peso el principio reeducativo en los procesos de privación de libertad (Añaños, 2012). A esto se añade la presencia cada vez mayor de educadores/as sociales y educadores/es o monitores/as socioculturales y otros perfiles profesionales similares en la plantilla penitenciaria. Todo ello, sin poder obviar la presencia de entidades externas colaboradoras que realizan un trabajo esencial en el contexto penitenciario. Según la Secretaría General de Instituciones Penitenciarias (2019) en los años 2018-2019 cuentan con la presencia de 876 ONGs y entidades colaboradoras que participan en la ejecución de 1.017 programas de intervención.

Todos ellos abordan, de una forma u otra, cuestiones relacionadas con la generación y el mantenimiento de relaciones dentro de prisión. Estudios previos sobre esta temática ponen de manifiesto cómo las relaciones reducen la ansiedad y la incertidumbre que produce la situación en la que se encuentran las personas internas, además de ser útiles no solo para obtener información sobre el funcionamiento de un contexto extraño, ganar influencia entre las personas internas y entre los funcionarios sino para desarrollar un sentimiento de seguridad (Bayón et al., 1999; Crewe, 2009; Goffman, 1987; Viegas, 2009). Se trata de relaciones diarias marcadas por el contexto y por el particular microclima social de la institución, que se configuran a partir del estatus social creado en ese entorno y de los roles que se ejercen en él; siendo relaciones claramente diferenciadas entre funcionarios, de éstos con los internos/as y entre las personas recluidas (Clemente, 1997; García-Vita y Melendro, 2013). De ahí que estas relaciones se tornen centrales en el análisis de este trabajo, con especial interés en el análisis de en qué medida se potencian en prisión y con el entorno para facilitar la reinserción.

\section{El acompañamiento profesional para la reinserción}

Una de las maneras más eficaces de conseguir generar unas relaciones positivas y un nivel óptimo de implicación del personal penitenciario es promoviendo un constante y bien estructurado acompañamiento a las personas que se encuentran condenadas en establecimientos penitenciarios, potenciando relaciones sanas, labor que cobra especial significado cuando hablamos de personas que están en un momento avanzado de la reinserción, en una modalidad de semilibertad.

El término acompañamiento desde la praxis profesional ha sido utilizado para hablar de diversas formas de entender y trabajar con personas que, normalmente, atraviesan situaciones de riesgo, dificultad, exclusión o requieren algún tipo de orientación, desde la proximidad y la constancia (Fabra et al., 2016). Sus usos entre los y las profesionales sociales incide en el paso de una labor asistencial a una basada en la solidaridad, entendiendo que la acción social permite resolver los problemas a través de la escucha, el apoyo y la ayuda mutua, promoviendo en las personas con necesidades sociales el desarrollo máximo de su autonomía y sus proyectos vitales (Planella, 2008). Es por esto que el acompañamiento tiene dos componentes claros: el establecimiento de una relación de confianza y estable con las personas a las que se acompaña, a la vez que se le da protagonismo a éstas en el proceso de cambio o resolución que deseen iniciar (Ruiz y Santibáñez, 2014). Así, si bien el acompañamiento puede y debe operativizarse y planificarse (Alonso y Funes, 2009), el mero hecho de estar presente y disponible para las personas es una herramienta valiosa, explicada por la percepción de contar con un apoyo (García-Vita y Melendro, 2013; Santibáñez, 2009).

De esta manera, el acompañamiento a personas condenadas a prisión es clave para su retorno a la vida en sociedad y la inclusión sociolaboral, familiar y comunitaria. En este caso, el acompañamiento cobra sentido desde la labor educativa y de reinserción. La acción de educar en el medio penitenciario 
es siempre de acompañamiento (Fabra et al., 2016; Ortega, 2010). Los y las profesionales penitenciarios y voluntarios/as, en gran medida, cuando hablamos de personas en semilibertad, se convierten en mediadoras entre el interno/a y la sociedad (Ortega y Hernández, 2015). Acompañar a un recluso o reclusa implica educar para la libertad y educar para la toma de decisiones y para que puedan retomar el protagonismo de sus vidas (Caride, 2010; Enjuanes y Vinyals, 2016; Ortega y Hernández, 2015). En ese proceso de acompañamiento en semilibertad también es indispensable la gestión de recursos externos a los centros penitenciarios, siendo esencial el trabajo de las entidades colaboradoras y el voluntariado (Enjuanes y Vinyals, 2016), así como con aquellas que ayuden a trabajar con las redes sociofamiliares del exterior (García-Vita et al., 2021).

Las mujeres viven su encarcelamiento de forma diferente, con mayores índices de sufrimiento sobre todo vinculado al sentir de que están desatendiendo sus obligaciones familiares, y esto debe considerarse al diseñar los programas y acciones penitenciaria con ellos específicamente (Yagüe, 2007). Las intervenciones centradas en el acompañamiento con mujeres reclusas han sido puestas en valor en trabajos como los de García-Vita y Melendro (2013), Malek y Puche (2012) o Yagüe (2007). Estas ponen en el centro de la misma al sujeto beneficiario, en este caso a la mujer, individualizando las acciones, partiendo de la propia persona y no de su yo delictivo, haciéndola protagonista de cada decisión, respetando su momento vital y sus propias circunstancias, así como teniendo en consideración sus circunstancias compartidas como es el ser mujer, madre o compañera (Ayuste y Payá, 2014). En concreto, para el caso de las mujeres condenadas a prisión, las situaciones previas al ingreso se unen a la insuficiente presencia de programas y acciones específicas se hace evidente, a la par que los resultados de experiencias de acompañamiento diseñados particularmente para ellas suelen tener un impacto positivo e incluso traspasar la esfera penitenciaria e incidir en sus proyectos de vida posteriores (Malek y Puche, 2012). Además, se requiere un ambiente de confianza y seguridad que enfatice las relaciones interpersonales positivas intramuros (García-Vita y Melendro, 2013; Yagüe, 2007).

\section{Metodología}

\section{Participantes}

La población participante fue el total de mujeres que se encontraban en tercer grado o segundo grado especial en proceso de semilibertad, en el medio abierto del sistema penitenciario español en el momento de realización del trabajo de campo; así como los profesionales encargados de intervenir con mujeres en dicha modalidad. La muestra está compuesta por 310 mujeres reclusas (30.1\% de la población total femenina), pertenecientes a 31 centros que representan los distintos recursos de cumplimento de condena en medio abierto (5 en entidades colaboradoras y 26 correspondientes a la institución penitenciaria, representando éstos el $63.3 \%$ del total de centros en medio abierto en todo el territorio nacional). En el caso de los profesionales se ha contado con una muestra de 66 participantes $(69.7 \%$ mujeres) que trabajaban en 16 provincias distintas de 10 comunidades autónomas. De ellos, un 33.3\% son psicólogos, un $1.8 \%$ trabajadores sociales, un $15.2 \%$ educadores sociales, un $9.1 \%$ pedagogos o maestros, un $6.1 \%$ técnicos terapéuticos, un $4.5 \%$ profesionales del ámbito sanitario y un $3 \%$ juristas o abogados.

\section{Instrumentos}

El estudio utiliza como instrumentos un cuestionario mixto destinado a mujeres reclusas, un cuestionario mixto destinado a profesionales del medio penitenciario y una entrevista semiestructurada, todos diseñados ad hoc. Específicamente en este trabajo se han analizado cinco preguntas del cuestionario de mujeres, concretamente las relativas a si han recibido apoyo o asesoramiento (pregunta 
cerrada de tipo dicotómica con respuestas "sí" o "no"), de qué tipo ha sido (pregunta abierta) y profesionales a los/as que acuden para realizar ese proceso de acompañamiento (pregunta con múltiples ítems que representan la diversidad de profesionales penitenciarios de respuesta dicotómica "sí" o "no"). También se han analizado dos preguntas del cuestionario a profesionales relativas a la valoración del proceso de acompañamiento que se desarrolla con las mujeres reclusas (pregunta de respuesta abierta) y el tipo o en qué consiste ese asesoramiento y cómo se ha llevado a cabo (pregunta de respuesta abierta).

\section{Procedimiento}

El trabajo está avalado y aprobado por los comités éticos de la Secretaría General de Instituciones Penitenciarias y la Consejería de Justicia de la Generalitat de Cataluña (única con competencias en materia penitenciaria). Para la obtención de la muestra de mujeres se ha seguido un muestreo bietápico: primero la elección de los centros según representación territorial y mayor ratio de mujeres y, segundo, elección al azar entre las mujeres que querían participar, siempre y cuando cumplieran el requisito de haber pasado previamente por la reclusión en prisión en régimen ordinario. El margen de error de los datos (nivel de confianza del 95\%) es de 4.5 puntos. En el caso de los profesionales, la muestra se obtuvo mediante un muestreo aleatorio simple entre los trabajadores del medio penitenciario que han querido participar. En todos los casos se ha obtenido el consentimiento informado por escrito de las mujeres y profesionales que conforman la muestra.

\section{Análisis de Datos}

La investigación ha empleado métodos cualitativos y cuantitativos, de forma complementaria. Se han analizado descriptivamente los resultados para valorar la opinión de las mujeres y los y las profesionales de la muestra durante el proceso de semilibertad, la tipología de acompañamiento y el grado de necesidad del mismo para su proceso de reinserción e inclusión social. Los datos cuantitativos han sido tratados en SPSS IBM versión 23 y 24, realizándose un análisis descriptivo de frecuencias. Los datos cualitativos relativos a las preguntas de respuesta abierta fueron categorizados a través del análisis de contenidos, generando categorías emergentes para proceder a su análisis.

\section{Resultados}

Las mujeres participantes en este estudio tienen una media de 42 años y 10 meses, con un mínimo de 20 y un máximo de 74 años y una desviación estándar de 10.68. Según rangos de edad $24.8 \%$ entre los 26 y 35 años, el $35.8 \%$ se encuentra entre los 36 y los 45 años, y el 29.4\% entre los 46 y los 60 años. En cuanto al tiempo de estancia en prisión la media (sumando todos los ingresos que han podido tener a lo largo de sus vidas) es de 4 años y 2 meses, con un mínimo de 1 mes y un máximo de 24 años y 10 meses y una desviación estándar de 47.48. Según rangos de tiempos el 29.7\% lleva entre 2 y 5 años en prisión, el $20.6 \%$ entre 5 y 15 años y el $20 \%$ entre 1 y 2 años.

El análisis descriptivo de los resultados muestra, en primer lugar, cómo las mujeres encuestadas responden a si han tenido apoyo o asesoramiento durante su estancia en las distintos centros penitenciarios durante su condena de forma positiva, obteniendo un $85.2 \%(n=264)$ de respuestas afirmativas, frente a un $12.6 \%(n=39)$ de ellas que han contestado no haber tenido asesoramiento durante todo su proceso.

En la actualidad, en semilibertad, en el centro que están en ese momento de la condena, reconocen estar recibiendo ayuda un 31.3\% $(n=97)$ de las mujeres frente a un $19 \%(n=59)$ que afirman no estar recibiéndola. El resto, $49.7 \%(n=154)$ no han contestado a la pregunta. 
En la pregunta sobre qué tipo de ayuda están recibiendo, las respuestas de tipo cualitativo han sido categorizadas, a pesar de que el $74.9 \%(n=232)$ no han respondido a la pregunta bien por no haber recibido ayuda de este tipo o bien por haberla dejado en blanco. Los resultados muestran que el $12.6 \%$ $(n=39)$ de las mujeres participantes afirman que el tipo de ayuda recibida es la intervención del equipo técnico, un 5.5\% $(n=17)$ mencionan ayuda de tipo económica, un 3.5\% $(n=11)$ dicen recibir apoyo externo de asociaciones y otro 3.5\% $(n=11)$ señalan que la ayuda es de tipo orientación formativa, laboral o material. El resto no ha respondido a la pregunta.

En una pregunta de tipo dicotómica -en la que todas las participantes debían responder sobre cada profesional- sobre a quién acuden para pedir ayuda, un 54.2\% $(n=168)$ reconocen acudir al trabajador/a social, el 46.8\% $(n=145)$ de los casos lo hacen a educadores/a, un $32.9 \%(n=102)$ a un/a psicólogo/a, un 19.4\% $(n=60)$ lo hace a un funcionario de interior. Los porcentajes disminuyen con respecto a la figura de los y las juristas, figura a la que acuden un $16.8 \%(n=52)$ de la muestra. Seguido de un $9.7 \%(n=30)$ que acude a asociaciones sin ánimo de lucro.

Tabla 1.

Profesionales a los que acuden las mujeres en semilibertad a solicitar ayuda o apoyo

\begin{tabular}{lcccccccc} 
& \multicolumn{3}{c}{ Sí } & \multicolumn{2}{c}{ No } & \multicolumn{2}{c}{ En blanco } & \multicolumn{2}{c}{ Totales } \\
\cline { 2 - 9 } & $n$ & $\%$ & $n$ & $\%$ & $n$ & $\%$ & $n$ & $\%$ \\
\hline 1. Educador & 145 & 46.8 & 157 & 50.6 & 8 & 2.6 & 310 & 100 \\
\hline 2. Trabajador/a social & 168 & 54.2 & 134 & 43.2 & 8 & 2.6 & 310 & 100 \\
\hline 3. Psicólogo/a & 102 & 32.9 & 200 & 64.5 & 8 & 2.6 & 310 & 100 \\
\hline 4. Jurista & 52 & 16.8 & 248 & 80 & 10 & 3.2 & 310 & 100 \\
\hline 5. Terapeuta & 18 & 5.8 & 282 & 91 & 10 & 3.2 & 310 & 100 \\
\hline 6. Figura religiosa & 26 & 8.4 & 275 & 88.7 & 9 & 2.9 & 310 & 100 \\
\hline 7. Voluntariado de asociaciones/ONG & 30 & 9.7 & 270 & 87.1 & 9 & 2.9 & 310 & 100 \\
\hline 8. Maestro & 29 & 9.4 & 272 & 87.7 & 9 & 2.9 & 310 & 100 \\
\hline 9. Funcionario de interior & 60 & 19.4 & 232 & 74.8 & 9 & 2.9 & 310 & 100 \\
\hline 10. Otros & 39 & 12.6 & 0 & 0 & 271 & 87.4 & 310 & 100 \\
\hline
\end{tabular}

Dentro del cuestionario a los profesionales, en la pregunta acerca de en qué consiste y cómo se está llevando a cabo el acompañamiento para la reinserción social con las mujeres en semilibertad, los y las encuestados/as afirman, en un $28.8 \%(n=19)$, que se atiende de forma individualizada a sus necesidades. Mientras que, un 13.6\% $(n=9)$ responden que el acompañamiento se deriva o se da a través de programas integrales y formación.

\section{Tabla 2.}

Tipos de acompañamiento profesional para la reinserción social de mujeres en semilibertad

\begin{tabular}{lcc} 
& $n$ & $\%$ \\
\hline Recursos externos, derivación & 9 & 13.6 \\
\hline Programa integral, formación & 9 & 13.6 \\
\hline Empleo: inserción laboral & 5 & 7.6 \\
\hline Atención individualizada de necesidades & 19 & 28.8 \\
\hline Otros & 5 & 7.6 \\
\hline En blanco & 19 & 28.8 \\
\hline Total & 66 & 100 \\
\hline
\end{tabular}


Con respecto a la valoración sobre el proceso de acompañamiento, el 13.6\% $(n=9)$ de los profesionales reconocen como más necesaria la atención individualizada, frente a un $10.6 \%(n=7)$ que afirma la necesidad de recursos humanos y materiales.

Tabla 3.

Valoración de los profesionales sobre el acompañamiento profesional a mujeres en semilibertad

\begin{tabular}{lcc} 
& $n$ & $\%$ \\
\hline Atención individualizada & 9 & 13.6 \\
\hline Eficaz en la consecución de objetivos & 5 & 7.6 \\
\hline Falta de recursos humanos y/o materiales & 7 & 10.6 \\
\hline otros & 5 & 7.6 \\
\hline En blanco & 39 & 59.1 \\
\hline No procede & 1 & 1.5 \\
\hline Total & 66 & 100 \\
\hline
\end{tabular}

\section{Discusión}

Construir un ambiente idóneo entre reclusas y profesionales e implicar a todo el personal que trabaja con las internas son estrategias básicas de la intervención penitenciaria (Del Rey, 2004; GarcíaVita y Melendro, 2013). De los datos de la investigación se puede destacar como primer resultado que las internas cuentan con asesoramiento en prisión. Como no podía ser de otra manera en un contexto tan intervenido, la presencia de profesionales y voluntarios/as ocupa sus días. No obstante, esta presencia es desigual. Si bien, el equipo técnico aparece como aquel con quien más se cuenta, dentro de este son los y las trabajadoras sociales, con un $54.2 \%$, y los y las educadoras sociales, con un $46.8 \%$ de mujeres participantes, a quienes más acuden si necesitan ayuda. Este hecho coincide con los resultados de una investigación anterior (García-Vita y Melendro, 2013) donde se concluye que profesionales como los educadores/as y otros, muy frecuentes en la cotidianeidad penitenciaria, son con quienes mejores relaciones construyen y en quienes más se apoyan. Este dato se comprende desde la perspectiva de que el estar presente y mostrarse disponible para las personas privadas de libertad es una herramienta relevante en el acompañamiento profesional (García-Vita y Melendro, 2013; Santibáñez, 2009). Así mismo, entronca con la historia de ambas disciplinas. El Trabajo Social, con más tradición en este contexto, se viene ocupando de las mujeres en prisión, contando con Concepción Arenal como uno de sus referentes principales. La Educación Social, con un recorrido más breve en el tiempo, toma un lugar protagónico también al entenderse el medio penitenciario como un espacio re-educador, tal y como decíamos antes. Así, no es de extrañar que estos/as sean los/as profesionales más demandados/as, frente a profesiones como el o la terapeuta, que no lo son tanto. También influyen en esta demanda tan desigual, la segmentación de los y profesionales en diferentes categorías, como si de "compartimentos estancos" se tratara, o las circunstancias particulares de cada una de las mujeres a las que se les ha preguntado.

Pero ¿de qué tipo de acompañamiento estamos hablando? Tal y como se muestra en el apartado anterior, fundamentalmente se trata de un acompañamiento individualizado. Esto es coincidente con el tipo de atención/intervención social que predomina en el sistema de servicios sociales español (ver algunos apuntes en este sentido en Valenzuela-Vela y Alcázar-Campos, 2020) donde lo grupal, y no digamos ya, lo comunitario, son relegados al olvido. Y coincide con la idea según la cual hay que promover el principio de actividad en la persona privada de libertad y extender la visión del cumplimiento de la condena como un tiempo de actividad muy controlado que aspira al cambio personal, 
y no un tiempo de pasividad despersonalizadora (Gil, 2010). Es decir, una de las máximas de la intervención en el medio penitenciario consiste en luchar contra la pasividad. Pasividad frente a la que, según las entrevistas hechas a los y las profesionales, se ponen en marcha distintos proyectos, bien basados en las necesidades concretas de las mujeres, abordadas de forma individual, bien mediante la puesta en marcha de programas integrales y formación. Estos programas se asientan en un concepto del tratamiento específico y complejo, con énfasis en la atención a colectivos vulnerables, como son las mujeres en el medio penitenciario, mediante la puesta en marcha de acciones para la igualdad que contrarresten la situación discriminatoria en el contexto penitenciario (Añaños-Bedriñana y Yagüe, 2013). Estas intervenciones plantean la obligación de la prisión de convertirse en una institución que trasmita una profunda mediación valorativa sobre los modos adecuados de conducirse en la vida, donde la formación es fundamental. Revalorizando la acción del interno/a (Almeda-Samaranch, 2017).

Tras los resultados obtenidos se puede concluir que el acompañamiento aquí resulta fundamental. Acompañamiento socioeducativo, entendido como un proceso en el que se facilita la identificación de un proceso de cambio personal, la reflexión, el apoyo y el empoderamiento personal. Donde la persona, el/la educador/a y la comunidad son agentes activos y comprometidos (Fabra et al., 2016). Donde las mujeres sean consideradas desde sus particularidades individuales y colectivas, familiares y sociales, laborales y económicas (Añaños-Bedriñana y Yagüe, 2013; Malek y Puche, 2012; Yagüe, 2007) como paso necesario para asegurar un proceso de acompañamiento socioeducativo adecuado y de impacto (Ayuste y Payá, 2014).

\section{Conclusiones, limitaciones y futuras líneas}

Somos conscientes de todo lo que queda por hacer y del déficit en investigación social sobre el proceso de acompañamiento. Así, esta investigación nos abre nuevos interrogantes: al margen de la necesidad de materiales y recursos humanos en la labor de acompañamiento, que se manifiesta en las entrevistas, ¿qué aspectos más serían necesarios? ¿qué sucede con la intervención grupal e, incluso con la comunitaria, en las tareas de acompañamiento? ¿se pueden/deben poner en funcionamiento planes integrales que conecten la intervención de los y las profesionales, al margen de sus funciones? ¿cómo se concretan, en los procesos de reinserción y salida de prisión, estos acompañamientos? Estas y otras preguntas nos siguen animando en nuestra tarea investigadora, convencidas de que solo mediante la acción socioeducativa lograremos apoyar los procesos de reinserción de quienes han pasado por prisión.

El desarrollo documental y de datos del presente artículo está bajo la supervisión y ejecución del proyecto "Procesos de reinserción y acompañamiento a mujeres en semilibertad" analizando para ello las variables objeto de estudio. Los datos obtenidos en el mismo, por lo tanto, está condicionado a la recogida de información puntual y no a la comparación entre diferentes momentos en la situación de las reclusas.

De la misma manera los datos son insuficientes con respecto al proceso de acompañamiento en lo que a literatura e investigación se refiere. Dejando un margen de contraste y discusión de información deficitario, lo que abre un campo de trabajo para investigaciones posteriores

\section{Agradecimientos}

Este trabajo se enmarca en el Proyecto de Investigación I+D+I -REINAC- "Procesos de reinserción y acompañamiento a mujeres en semilibertad", Ref. EDU2016-79322-R (2016-2020). Financiado por el Plan Nacional de Investigación, Proyectos de Investigación I+D+I, Ministerio de Economía y Competitividad (MINECO), Agencia Estatal de Investigación (AEI) y FEDER (España). 


\section{Referencias}

Almeda-Samaranch, E. (2017). Criminologías feministas, investigación y cárceles de mujeres en España. Papers Revista de Sociología, 102(2), 151-181. http://dx.doi.org/10.5565/rev/papers.2334

Alonso, I. y Funes, J. (2009). El acompañamiento social en los recursos socioeducativos. Educación Social. Revista de Intervención Socioeducativa, 42, 28-46.

Añaños-Bedriñana, F. T. (2010). Mujeres presas y su relación con las drogas. Implicaciones desde la Educación Social. En F. T. Añaños-Bedriñana (coord.), Las mujeres en las prisiones: la Educación Social en contextos de riesgo y conflicto (pp. 77-100). Gedisa.

Añaños-Bedriñana, F. T. (2012). Violencias y exclusiones en el medio penitenciario. Enfoque socioeducativo y de la paz. Convergencia, Revista de Ciencias Sociales, 19(59), 13-51.

Añaños-Bedriñana, F. T., y Yagüe, C. (2013). Presentación: Educación Social en prisiones. Planteamientos iniciales y políticas encaminadas hacia la reinserción desde la perspectiva de género. Pedagogía Social. Revista Interuniversitaria, 22, 7-12.

Ayuso, A. (2001). La intervención socioeducativa en el tratamiento penitenciario. Pedagogía Social. Revista Interuniversitaria, 6-7, 73-99.

Bayón, F., Compadre, A., y Salarich, L. (1999). La integración social de los exreclusos. En M. Clemente y M. I. Serrano (coords.), Psicología Jurídica y Redes Sociales, (pp. 375-413). Fundación Universidad-Empresa.

Caride, J. A. (2010). La Educación Social como práctica de y hacia la libertad en contextos penitenciarios. En Fanny T. Añaños-Bedriñana (coord.), Las mujeres en las prisiones: la Educación Social en contextos de riesgo y conflicto (pp. 45- 64). Gedisa.

Clemente, M. (1997). La organización social informal en la prisión. En M. Clemente y J. Núñez (coords.), Psicología Jurídica Penitenciaria II (pp. 321-356). Fundación Universidad Empresa.isép.

Crewe, B. (2009). The Prisoner Society: Power, Adaptation, and Social Life in an English Prison. Oxford University Press. [i]

Del Pozo, F. J., y Añaños-Bedriñana, F. T. (2013). La Educación Social Penitenciaria ¿De dónde venimos? Y ¿hacia dónde vamos? Revista Complutense de Educación, 24(1), 47-68.

Del Rey, E. (2004). Intervención ambiental con drogodependientes encarcelados: principios, datos y líneas de actuación. Revista de Estudios Penitenciarios, 250, 73-96.

Enjuanes, J., y Vinyals, O. (2016). Voluntariado y educación social en prisiones. Una experiencia de participación integral en el proceso de cambio de las personas privadas de libertad. Revista de Educación Social, 22, 212-229.

Fabra, N., Heras, P., y Fuertes, S. (2016). La reinserción social postpenitenciaria: un reto para la Educación Social. Revista de Educación Social, 22, 143-157.

García-Vita, M. M. (2016). Redes de apoyo y entornos sociofamiliares en mujeres reclusas: Análisis de las relaciones con las drogas, el acompañamiento en prisión y los procesos hacia la reinserción social. [Tesis doctoral, Universidad de Granada]. http://0hera.ugr.es.adrastea.ugr.es/tesisugr/26082275.pdf

García-Vita, M. M., y Melendro Estefanía, M. (2013). El ambiente en prisión: la atención recibida por las reclusas y las relaciones intramuros. Pedagogía Social. Revista Interuniversitaria, 22, 43-56.

García-Vita, M. M., Rivera, M., Raya, R., y Amaro, A. (2021). Análisis del grado y tipo de apoyo familiar a mujeres en semilibertad en España. Psychology, Society \& Education, 13(1), 61-71.

Gil, F. (2010). La acción pedagógica en las prisiones. Posibilidades y límites. Revista Española de Pedagogía, 245, 49-64.

Goffman, E. (1987). Internados. Amorrortu.

Malek, S., y Puche, I. (2012). Experiencia de Intervención con Mujeres Privadas de Libertad en Etapa 
de Pre-Egreso. Revista Electrónica de Psicología Social Poiésis FUNLAM, 12(24), 1-11.

Ortega, P. (2010). Educar es responder a la pregunta del otro. Edetania, 37, 13-31.

Ortega, P., y Hernández, M. A. (2015). La acción socioeducativa del voluntario en una prisión. Edetania, 47, 109-128.

Planella, J. (2008). Educación social, acompañamiento y vulnerabilidad: hacia una antropología de la convivencia. Revista Iberoamericana de Educación, 46(5), 1-14.

Redondo, S. (2004). Tratamiento y Sistema Penitenciario. En José Sanmartín (coord.), El laberinto de la violencia: causas, tipos y efectos (pp. 331-341). Ariel.

Redondo, S., Pozuelo, F., y Ruiz, A. (2007). El tratamiento en las prisiones: Investigación internacional y su situación en España. En Ana Isabel Cerezo y Elisa García (coords.), La prisión en España: una perspectiva criminológica (pp. 175-212). Comares.

Ruiz, M., y Santibáñez, R. (2014). Prisión, drogas y educación social. Educación Social. Revista de Intervención Socioeducativa, 57, 118-134.

Santibáñez, R. (2004). Exclusión y ciudadanía. Los retos de la pedagogía social. En F. Burrows (Comp.), Caminos para la ciudadanía: Actas del Congreso de Participación Ciudadana, Pedagogía Social y Desarrollo Local de Villarrica, Chile.

Secretaría General de Instituciones Penitenciarias (2014). El sistema penitenciario español. Ministerio del Interior, Secretaría General Técnica.

Secretaría General de Instituciones Penitenciarias (2018). Informe General 2018. Ministerio del Interior, Secretaría General Técnica.

Valenzuela-Vela, L., y Alcázar-Campos, A. (2020). Gendered Carceral Logics in Social Work: The Blurred Boundaries in Gender Equality Policies for Imprisoned and Battered Women in Spain. Affilia. Women and Social Work, 35(1), 73-88.

Viegas, F. (2009). La reja en la cabeza. Etnografía, representaciones, experiencias y mediaciones en torno al impacto de la cárcel en personas con libertad ambulatoria. Revista Question, 1(23). 